Document downloaded from:

http://hdl.handle.net/10251/148003

This paper must be cited as:

Llorens Rodríguez, R.; Borrego, A.; Palomo, P.; Cebolla, A.; Noé-Sebastián, E.; Bermúdez I Badia, S.; Baños Rivera, RM. (2017). Body schema plasticity after stroke: Subjective and neurophysiological correlates of the rubber hand illusion. Neuropsychologia. 96:61-69. https://doi.org/10.1016/j.neuropsychologia.2017.01.007

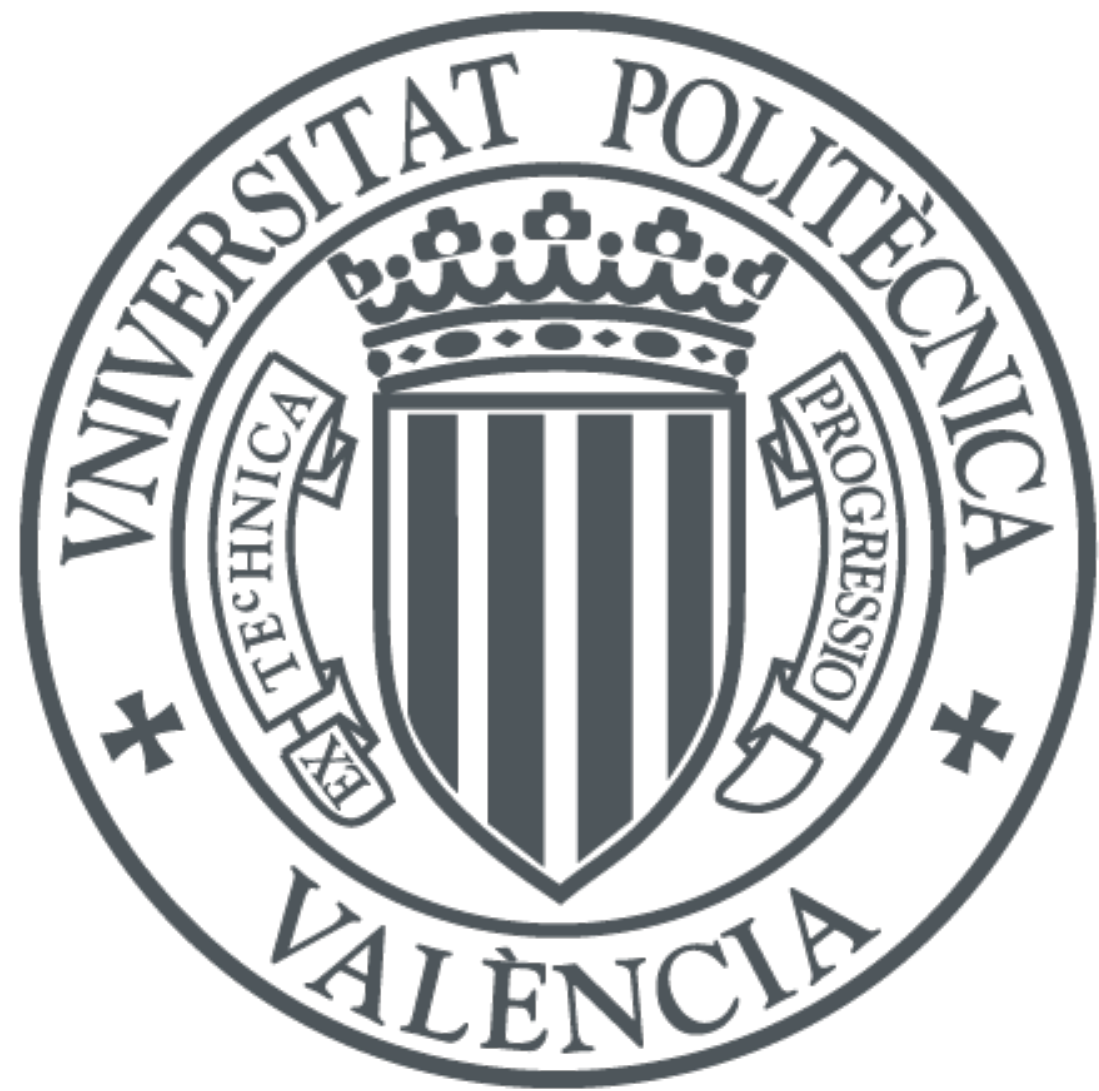

The final publication is available at

https://doi.org/10.1016/j.neuropsychologia.2017.01.007

Copyright Elsevier

Additional Information 


\title{
Body Schema Plasticity after Stroke: Subjective and Neurophysiological Correlates of the Rubber Hand Illusion
}

\author{
Roberto Llorens ${ }^{1,2}$, Adrián Borrego ${ }^{1}$, Priscila Palomo ${ }^{3}$, Ausiàs Cebolla ${ }^{3,4}$, Enrique Noé ${ }^{2}$, \\ Sergi Bermúdez i Badia ${ }^{5}$, Rosa Baños ${ }^{3,4}$ \\ ${ }^{1}$ Neurorehabilitation and Brain Research Group, Instituto de Investigación e Innovación \\ en Bioingeniería, Universitat Politècnica de València, Camino de Vera s/n, 46022 \\ Valencia, Spain. \\ ${ }^{2}$ Servicio de Neurorrehabilitación y Daño Cerebral de los Hospitales NISA, Fundación \\ Hospitales NISA, Río Tajo 1, 46011 Valencia, Spain. \\ ${ }^{3}$ Universitat de València, Av. Blasco Ibáñez, 13, 46010 Valencia, Spain. \\ ${ }^{4}$ Fisiopatología de la Obesidad y la Nutrición (CIBERobn), Madrid, Spain. \\ ${ }^{5}$ Madeira-ITI, Universidade da Madeira, Campus universitario da Penteada, 9020-105 \\ Funchal, Portugal.
}

\section{Correspondence:}

Dr. Roberto Llorens

Universitat Politècnica de València

Camino de Vera s/n, 46022

Valencia, Spain.

Tel: +3496387751

rllorens@1ableni.com

Pages: 23

Number of figures: 3

Number of tables: 5 


\begin{abstract}
Stroke can lead to motor impairments that can affect the body structure and restraint mobility. We hypothesize that brain lesions and their motor sequelae can distort the body schema, a sensorimotor map of body parts and elements in the peripersonal space through which human beings embody the reachable space and ready the body for forthcoming movements. Two main constructs have been identified in the embodiment mechanism: body-ownership, the sense that the body that one inhabits is his/her own, and agency, the sense that one can move and control his/her body. To test this, the present study simultaneously investigated different embodiment subcomponents (bodyownership, localization, and agency) and different neurophysiological measures (galvanic skin response, skin temperature, and surface electromyographic activity), and the interaction between them, in clinically-controlled hemiparetic individuals with stroke and in healthy subjects after the rubber hand illusion. Individuals with stroke reported significantly stronger body-ownership and agency and reduced increase of galvanic skin response, skin temperature, and muscular activity in the stimulated hand. We suggest that differences in embodiment could have been motivated by increased plasticity of the body schema and pathological predominance of the visual input over proprioception. We also suggest that differences in neurophysiological responses could have been promoted by a suppression of the reflex activity of the sympathetic nervous system and by the involvement of the premotor cortex in the reconfiguration of the body schema. These results could evidence a body schema plasticity promoted by the brain lesion and a main role of the premotor cortex in this mechanism.
\end{abstract}

\title{
Keywords
}

Body schema, Body-ownership, Embodiment, Rubber hand illusion, Stroke, Neurophysiology

\author{
Abreviations \\ RHI: Rubber Hand Illusion; GSR: Galvanic Skin Response; sEMG: surface \\ Electromyographic Activity
}




\section{Introduction}

Embodiment is a multi-component psychological construct that has been explained as the sense of one's own body (Arzy, Overney, Landis, \& Blanke, 2006), as the bodily self-consciousness (Legrand, 2006), or as corporeal awareness (Berlucchi \& Aglioti, 1997). Although different definitions have been proposed, embodiment could be understood as the representation of an element (bodily or not) within the body schema (de Vignemont, 2011). Recent research has focused on unifying aspects of the embodied cognition theories and on identifying its subcomponents, such as bodyownership and agency (Kalckert \& Ehrsson, 2012; Longo, Schüür, Kammers, Tsakiris, \& Haggard, 2008). Body-ownership can be defined as the sense that the body that one inhabits is his/her own (Tsakiris, 2010). Agency refers to the sense that one can move and control his/her body (Tsakiris, 2010). Consequently, body-ownership should be continuous and omnipresent and, in contrast, only voluntary actions, fired by efferent signals, should elicit agency. Both constructs have been postulated as dissociated concepts (Kalckert \& Ehrsson, 2012; Tsakiris, Longo, \& Haggard, 2010), but this is still a matter of debate (Ma \& Hommel, 2015).

The Rubber Hand Illusion (RHI) is an experiment that allows investigating body-ownership in the absence of movement (Botvinick \& Cohen, 1998). This phenomenon involves cross-modal interaction of sight, touch, and proprioception to create a convincing feeling of body-ownership over an external body part (Lloyd, Gillis, Lewis, Farrell, \& Morrison, 2013). Although this experiment has been widely replicated in neuroscience studies to determine the influence of sensory inputs on body representation (Ramakonar, Franz, \& Lind, 2011), the neural signatures of this illusion still remain unclear. Preliminary research points to the right insula (Tsakiris, Hesse, Boy, Haggard, \& Fink, 2007; Tsakiris et al., 2010), the posterior cingular cortex (Guterstam, Bjornsdotter, Gentile, \& Ehrsson, 2015), and the premotor cortex (Bekrater-Bodmann et al., 2014; Gentile, Bjornsdotter, Petkova, Abdulkarim, \& Ehrsson, 2015; Limanowski \& Blankenburg, 2015; Petkova et al., 2011; Zeller, Gross, Bartsch, Johansen-Berg, \& Classen, 2011) as having a major role in the RHI. Additionally, many different studies have investigated the underlying neurophysiological correlates of the phenomenon under different conditions, mainly examining variations in the skin temperature (Hohwy \& Paton, 2010; Kammers, Rose, \& Haggard, 2011; Moseley, Olthof, et al., 2008; Rohde, Wold, Karnath, \& Ernst, 2013; Salomon, Lim, Pfeiffer, Gassert, \& Blanke, 2013; Thakkar, Nichols, McIntosh, \& Park, 2011; van Stralen et al., 2014), and the Galvanic Skin Response (GSR) (Armel \& Ramachandran, 2003; D'Alonzo \& Cipriani, 2012; Ehrsson et al., 2008; Ma \& Hommel, 2013; Reinersmann et al., 2013), which represent autonomic nerve responses to the sweat gland function. However, these variables only reflect the function of the sympathetic nervous system. The influence of the RHI in other systems, and the interaction between these variables still remain unexplored.

Different studies have also assessed the effect of the RHI in different populations (Ehrsson et al., 2008; Reinersmann et al., 2013; Thakkar et al., 2011; van 
Stralen et al., 2014). The nature of stroke and its derived impairments could provide an interesting framework to study the embodiment constructs (de Vignemont, 2011). In fact, stroke has been posed to be a common cause of disorders of body schema (Corbett \& Shah, 1996), a neural representation of the body parts relative to each other and objects in the environment from the integration of visual, vestibular, and proprioceptive inputs (Corbett \& Shah, 1996; Haggard \& Wolpert, 2005). Motor impairments such as hemiparesis, a consequence of injuries to the pyramidal tract above the medulla that shows up as muscle weakness during voluntary movements in $50 \%$ of stroke survivors six months after onset (Kelly-Hayes et al., 2003), could exacerbate the incidence of these disorders. However, literature about the effect of stroke on embodiment is scant and to date few empirical studies exist. A big study involving also healthy participants initially reported that individuals with stroke were less likely to feel the illusion (Zeller et al., 2011). In contrast, more recent RHI studies in a subject with hand disownership (van Stralen, van Zandvoort, Kappelle, \& Dijkerman, 2013) and in pure hemiplegic subjects (Burin et al., 2015) showed stronger illusion on the affected hand than in the less affected hand, which could evidence an impaired sense of ownership or a tendency to gain ownership over external body parts in the hemiparetic side. More studies controlling the clinical variables that may affect the results and including neurophysiological recordings are needed to confirm this hypothesis and to elaborate a common rationale for the underlying neural processes that promote these effects. In addition, although a few attempts have been made to assess body-ownership after stroke, other sub-components of embodiment have been ignored.

We hypothesize that the functional and neurophysiological alterations derived from stroke would promote alterations in the body schema that would facilitate an intensification of the embodiment sub-components during the RHI compared to healthy individuals. We conjecture, in light of the existing evidence that supports the involvement of the premotor cortex in the brain mechanisms of the illusion, that the experiment would elicit different neurophysiological responses in both groups, not only in the skin temperature and the GSR but also in the muscular activity. The aim of this study is therefore to investigate the subjective (body-ownership, localization, and agency) and neurophysiological responses (skin temperature, GSR, and electromyography) to the RHI, and the relationship between them, in healthy subjects and clinically-controlled individuals with stroke.

\section{Methods}

\section{Participants}

Both hemiparetic individuals following a first time stroke and healthy subjects were recruited. Inclusion criteria in the stroke group were 1) age $\geq 50$ and $\leq 80$ years old; 2) chronicity > 6 months; 3) absence of severe cognitive impairment as defined by MiniMental State Examination (Folstein, Folstein, \& McHugh, 1975) > 23; and 4) able to follow instructions as defined by the receptive language subscale of the Mississippi Aphasia Screening Test (Romero et al., 2012) $\geq 45$. Individuals were excluded if they had 1) increase in muscle tone as defined by Modified Ashworth Scale (Bohannon \& 
Smith, 1987) > 3; 2) joint stiffness that prevented arm positioning according to the requirements of the study; 3) peripheral nerve damage affecting the upper extremities; 4) orthopedic alterations or pain syndrome of the upper limbs; 5) visual or hearing impairment that did not allow possibility of interaction; 6) unilateral spatial neglect; and 7) asomatognosia. Individuals with stroke were recruited from the total pool of outpatients who had suffered a stroke and were attending a long-term rehabilitation program in the Brain Injury Service of NISA Hospital Valencia al Mar (Valencia, Spain). Participants were included in the healthy group if they were 50 to 80 years old and had no motor or cognitive impairment. These participants were recruited using advertisements on social media and community outreach.

Twenty individuals with stroke and 21 healthy individuals satisfied inclusion and exclusion criteria and accepted to participate in the study (Table 1). After inclusion in the study, motor impairment of the participants with stroke was assessed with the Motricity Index (Kopp et al., 1997), and their sensory impairment in the hand and wrist was assessed with the Nottingham Sensory Assessment (Lincoln, Jackson, \& Adams, 1998), a standardized tool for multi-modal sensory examination that evaluates tactile sensation (light touch, pressure, pinprick sensation, temperature discrimination, tactile localization, and bilateral simultaneous stimulation), kinesthesia, and stereognosis. Handedness of healthy participants was assessed using the Edinburgh Handedness Inventory (Oldfield, 1971), as in previous studies (Ocklenburg, Ruther, Peterburs, Pinnow, \& Gunturkun, 2011).

Table 1. Characteristics of the participants

\begin{tabular}{|c|c|c|c|}
\hline & $\begin{array}{l}\text { Post-stroke } \\
\text { individuals } \\
\quad(n=20)\end{array}$ & $\begin{array}{c}\text { Healthy } \\
\text { individuals } \\
(n=21)\end{array}$ & Significance \\
\hline $\operatorname{Sex}(n, \%)$ & & & $\mathrm{p}=0.017$ \\
\hline Male & $15(75 \%)$ & $13(62 \%)$ & \\
\hline Female & $5(25 \%)$ & $8(38 \%)$ & \\
\hline Age (years) & $59.5 \pm 8.9$ & $59.9 \pm 7.5$ & $\begin{array}{c}\mathrm{NS} \\
(\mathrm{p}=0.845)\end{array}$ \\
\hline Chronicity (days) & $844.3 \pm 312.7$ & - & \\
\hline \multicolumn{4}{|l|}{ Etiology $(\mathrm{n}, \%)$} \\
\hline Hemorrhagic & $9(45 \%)$ & - & \\
\hline Ischemic & $11(55 \%)$ & & \\
\hline TACI & 4 & & \\
\hline PACI & 7 & & \\
\hline \multicolumn{4}{|c|}{ Laterality of the lesion $(\mathrm{n}, \%)$} \\
\hline Left & $10(50 \%)$ & - & \\
\hline Right & $10(50 \%)$ & & \\
\hline \multicolumn{4}{|c|}{ Non-dominant/hemiparetic side (n, \%) } \\
\hline Left & $10(50 \%)$ & $16(76 \%)$ & NS \\
\hline Right & $10(50 \%)$ & $5(24 \%)$ & $(\mathrm{p}=0.082)$ \\
\hline
\end{tabular}




\begin{tabular}{lcl}
\hline Modified Ashworth Scale [0-4] & $1.6 \pm 0.8$ & - \\
\hline Motricity Index [0-100] & & \\
$\quad$ Hemiparetic arm & $54.3 \pm 14.9$ & - \\
$\quad$ Non-hemiparetic arm & 100 & \\
\hline Nottingham Sensory Assessment & $1.2 \pm 0.8$ & - \\
$\quad$ Tactile sensation [0-2] & $0.9 \pm 0.9$ & \\
$\quad$ Kinesthesia [0-3] & $0.5 \pm 0.8$ & \\
$\quad$ Stereognosis [0-2] & $27.2 \pm 2.2$ & - \\
\hline Mini-Mental State Examination [0-30] & $49.0 \pm 1.6$ & - \\
\hline Mississippi Aphasia Screening Test [0- & & \\
50] &
\end{tabular}

Results of age, chronicity and clinical scales are expressed in terms of mean and standard deviation. Scores in the Modified Ashworth Scale of $1+$ were converted into 1.5 points. Comparisons between groups were performed with independent sample ttests (age) and Chi-squared (sex and non-dominant/hemiparetic side). TACI: Total Anterior Circulation Infarct; PACI: Partial Anterior Circulation Infarct.

The experimental protocol was approved by the Institutional Review Board of NISA Hospitals. All of the participants provided written informed consent prior to enrollment in the study.

\section{Materials}

The experiment was performed in a quiet room free of distractors where a conventional table $(120 \times 60 \times 75 \mathrm{~cm})$ with a movable wooden vertical board $(50 \times 40 \times 4 \mathrm{~cm})$ was arranged. Instrumentation included a man's and a woman's right and left rubber hand with forearm, two equal small brushes with a head diameter of $0.5 \mathrm{~cm}$, and a hammer. In addition, a black oversized tee was used to cover participants' both arms down to just above the forearms. A hole was made to introduce the arm of the rubber hand during the experiment.

Sensorization included a wearable wireless bracelet that measured the GSR and skin temperature (Affectiva®, Waltham, MA, USA), two extra sensors that also estimated the GSR (Twente Medical Systems International B.V., Oldenzaal, Netherlands), and two $\mathrm{Ag}-\mathrm{AgCl}$ sensors to estimate the surface electromyographic activity (sEMG) (Twente Medical Systems International B.V., Oldenzaal, Netherlands). A fake bracelet was handcrafted and fixed to the rubber hand to simulate the real one.

\section{Procedure}

Two experimenters conducted all of the sessions. Participants were blind to the purpose of the experiment. Initially, a brief description of the experiment, stimuli, and equipment was provided by Experimenter A. Experimenter B, who was equipped with the sensors between participants, helped participants to wear the oversized tee and equipped them with the sensors. The two GSR sensors were attached to the medial phalange of the index finger and the middle finger of the unstimulated arm, as in previous studies (Ehrsson et al., 2008; Ma \& Hommel, 2013). The wearable bracelet, 
which leaves the fingers free to be stimulated was fixed to the stimulated arm to also determine the responses in this side. The electromyographical sensors were fixed on the palmar (anterior) side of the forearm, one in the middle point of the distal third and the other between the middle and the proximal third of the arm under study. Sensors aimed to record the electrical activity mainly produced by the flexor muscles of the wrist and the fingers (palmaris longus, flexor carpis radialis, flexor digitorum superficialis, and pronator teres). Participants were required to sit in one side of the table in a comfortable position with both arms resting on the table and palms facing downward. Experimenter A sat in front of the participants and ask them to relax and maintain the position for 10 minutes for temperature acclimation and skin conductance stabilization. After that, the experimenter placed the vertical board in front of their right or left shoulder to hide the arm under study from sight (Figure 1). The rubber hand was placed in the other side of the frame at $15 \mathrm{~cm}$ to the participant's real hand (measured between index fingers) (Aimola Davies \& White, 2013; Kammers, de Vignemont, Verhagen, \& Dijkerman, 2009 ) and at $5.5 \mathrm{~cm}$ of the wooden frame, in an anatomically congruent position. The proximal end of the forearm of the rubber hand was covered with the tee to prevent the participants from seeing that it was not connected to their body, which might have reduced the vividness of the illusion (Ocklenburg et al., 2011).

Participants were asked to "pay attention to the rubber hand", and the experiment began. The participants' hand and forearm, and the rubber hand and forearm were synchronously brushed on the dorsal surface with the paintbrushes. Stimulation was administered in the hemiparetic side in participants with stroke and in the nondominant side in healthy individuals to match the reduced dexterity that individuals with stroke presented in the arm under study. Brush strokes were made at approximately $1 \mathrm{~Hz}$ in a proximal to distal direction with an unpredictable starting point (Kammers et al., 2011; Longo et al., 2008; Rohde et al., 2013). The length of the strokes was variable, ranging from $2 \mathrm{~cm}$ to $10 \mathrm{~cm}$, approximately. Stroking was applied for 120 seconds. After that, Experimenter B smashed the back of the rubber hand with the hummer and the experiment concluded. The experiment was conducted only once per participant. During the study, Experimenter B was in charge of the collection of the neurophysiological data.

\section{Figure 1. Experimental setting}
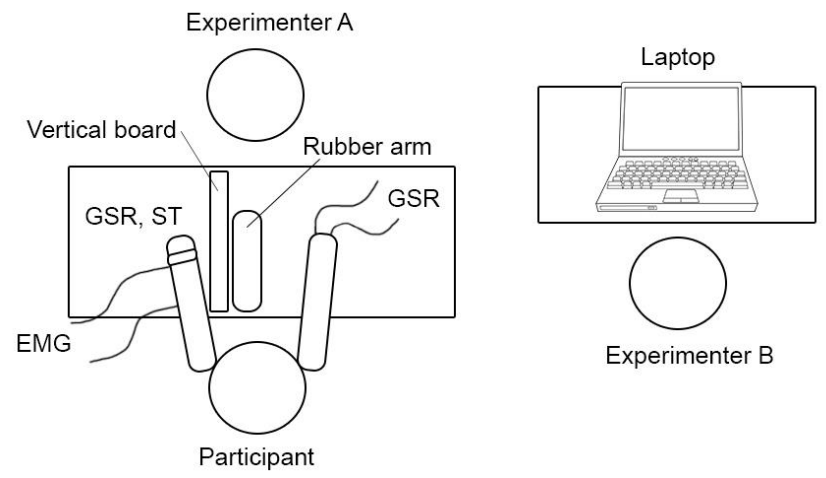
The picture describes the experimental setting of the experiment and the acquisition of the neurophysiological data. GSR: Galvanic skin response. ST: skin temperature. EMG: electromyography.

\section{Outcome measures}

After the experiment, participants were asked to indicate the extent of their agreement with ten statements about embodiment of the rubber hand. The questionnaire evaluated the extent that the rubber hand belonged to the participant, the participant had control over the rubber hand, the rubber hand and real hand were in the same location, and the rubber hand had taken on features of the actual hand (Longo et al., 2008) (Table 2). Participants rated the statements in a 7-item Likert scale, where a response of +3 indicated strongly agreement and -3 indicated strong disagreement. Before completing the questionnaire, the items were explained to the participants by a speech and language therapist.

Neurophysiological examination included variation in the GSR of both arms, and variation in the skin temperature and sEMG of the unstimulated arm.

\section{Table 2. Questionnaire about embodiment}

\section{Statements}

It seemed like I was looking directly at my own hand, rather than at a rubber hand

It seemed like the rubber hand began to resemble my real hand

It seemed like the rubber hand belonged to me

It seemed like the rubber hand was my hand

It seemed like the rubber hand was part of my body

It seemed like my hand was in the location where the rubber hand was

It seemed like the rubber hand was in the location where my hand was

It seemed like the touch I felt was caused by the paintbrush touching the rubber hand

It seemed like I could have moved the rubber hand if I had wanted

It seemed like I was in control of the rubber hand

Statements of the questionnaire about embodiment

\section{Data analysis}

The variation in the GSR was estimated as the difference between the maximum peak that occurred between 1 and $5 \mathrm{~s}$ after the hammer smash, and the mean value in the second previous to the smash, as described in previous works (Armel \& Ramachandran, 2003; Ma \& Hommel, 2013; Reinersmann et al., 2013). Only those variations greater than $0.03 \mathrm{mS}$ were considered meaningful (Armel \& Ramachandran, 2003; Reinersmann et al., 2013). The variation in the skin temperature was estimated as the difference between the mean temperature in the following $5 \mathrm{~s}$ after the hammer smash and in the $5 \mathrm{~s}$ previous to the stimulation, similarly to previous works (Rohde et al., 2013). Variation in the surface muscular activity was estimated as the difference between the averaged root mean square values of the sEMG signal in the $5 \mathrm{~s}$ previous to the stimulation and in the $5 \mathrm{~s}$ previous to the hammer smash. The signal was filtered 
using a band-pass filter (20-500 Hz, $48 \mathrm{~dB} /$ octave) and a notch filter $(50 \mathrm{~Hz}, 48$ $\mathrm{dB} /$ octave) (Huis In 't Veld, van Boxtel, \& de Gelder, 2014) and rectified for analysis (Clancy, Morin, \& Merletti, 2002). Artifacts in the signal caused by overt movement during these intervals were rejected. The neurophysiological data were processed offline using Matlab R2013b (MathWorks®, Natick, MA, USA).

Subcomponents of embodiment were defined according to the original description of the questionnaire as the average score of the first five statements (bodyownership), of the sixth to eighth statements (localization), and of the last two statements (agency) (Longo et al., 2008). Average scores greater than 0 were considered positive.

Given the number of observations of each outcome measure, their normality was checked using Shapiro-Wilk tests. Comparisons between the control and the experimental group in the scores of the questionnaire and in the neurophysiological data were performed with independent sample Mann-Whitney U tests. One-way analyses of covariance were performed to estimate the interaction between the demographical and clinical variables with the subjective and neurophysiological responses. Chi-square tests were performed to compare the percentage of participants from the two groups who experienced body-ownership. Spearman correlation analyses were performed between embodiment and neurophysiological measures. The $\alpha$ level was set at 0.05 for all analyses (two-sided). All analyses were computed with SPSS Statistics, version 22 (IBM®, New York, U.S.).

\section{Results}

\section{Embodiment}

Scores in the embodiment questionnaire showed that individuals with stroke felt a significantly stronger sense of body-ownership $(p=0.009)$ and agency $(p=0.046)$ than heathy individuals, while no significant differences were found in localization $(\mathrm{p}=0.656)$ (Table 3) (Figure 2). These results expressed in terms of number of participants who felt the effects also evidenced the differences between groups. While only 13 healthy participants $(61.9 \%)$ felt the sense of body-ownership, all of the participants with stroke but one $(95 \%)$ reported to have felt this effect $(\mathrm{p}=0.010)$. Similarly, only nine healthy participants $(42.9 \%)$ felt agency over the rubber hand in contrast to 16 participants with stroke $(80 \%)(\mathrm{p}=0.015)$. No demographical or clinical variable covaried with the reports of the participants with stroke in any embodiment component. In addition, participants with stroke who did not felt body-ownership or agency (Figure 2) also did not show differences neither in demographical nor clinical scales. 
Table 3. Results in the embodiment questionnaire

\begin{tabular}{lccc}
\hline & $\begin{array}{c}\text { Healthy group } \\
(\mathbf{n}=\mathbf{2 1})\end{array}$ & $\begin{array}{c}\text { Stroke group } \\
(\mathbf{n = 2 0})\end{array}$ & Significance \\
\hline Body-ownership & $0.9 \pm 1.9$ & $2.3 \pm 1.2$ & $\mathrm{p}=0.004$ \\
\hline Localization & $1.1 \pm 1.9$ & $0.9 \pm 0.9$ & $\mathrm{NS}(\mathrm{p}=0.266)$ \\
\hline Agency & $0.2 \pm 2.0$ & $1.6 \pm 2.2$ & $\mathrm{p}=0.009$ \\
\hline $\begin{array}{l}\text { Results are expressed in terms of mean and standard deviation. Scores range from }-3 \text { to } \\
+3 .\end{array}$ & &
\end{tabular}

Figure 2. Significant differences between groups in embodiment

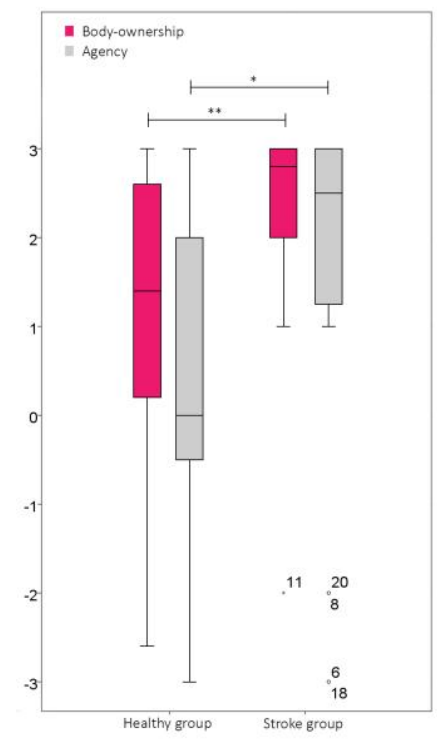

The picture shows $a$ box and whisker plot of body-ownership and agency. *: $p<0.05$; **: $p<0.001$.

\section{Electromyography}

Results evidenced significant differences between healthy subjects and participants with stroke ( $\mathrm{p}=0.005)$ (Table 4) (Figure 3). While the experiment caused an increase in the muscular activity of healthy subjects, participants with stroke experienced the opposite tendency, showing a decrease in the registered electromyographical data. Significant but smaller differences were also obtained when taking into account only those subjects who felt the sense of body-ownership (healthy participants experienced a variation of $0.91 \pm 3.07 \mathrm{mV}$, and participants with stroke of $-0.74 \pm 1.05 \mathrm{mV}$ ).

\section{Galvanic skin response}

All of the participants but one healthy subject increased their GSR in both hands during the experiment (Table 4) (Figure 3). Recordings in the unstimulated hand showed similar response in healthy subjects and individuals with stroke. However, recordings in the stimulated hand showed significantly different responses $(p=0.001)$. In terms of number of participants, all of the 13 healthy subjects who felt body-ownership 
experienced a meaningful increase on the GSR in the unstimulated hand, and 11 $(84.6 \%)$ in the stimulated hand. Similarly, all of the participants with stroke but one (94.7\%) experienced this increase in the unstimulated hand. However, this effect was detected in the stimulated hand only for four participants $(21.1 \%)$. Very similar results were detected when only those subjects who felt body-ownership were considered.

\section{Skin temperature}

All of the participants but three individuals with stroke experienced an increase in the average temperature, which was significantly higher in healthy subjects (Table 4) (Figure 3). When considering only those participants who experienced the sense of body-ownership, the rise was slightly higher in healthy subjects (from $0.70{ }^{\circ} \mathrm{C}$ to 0.82 ${ }^{\circ} \mathrm{C}$ ), while remained almost unaltered in participants with stroke (from $0.45^{\circ} \mathrm{C}$ to 0.47 $\left.{ }^{\circ} \mathrm{C}\right)$.

\section{Table 4. Neurophysiological data}

\begin{tabular}{lccc}
\hline & $\begin{array}{c}\text { Healthy group } \\
(\mathbf{n = 2 1})\end{array}$ & $\begin{array}{c}\text { Stroke group } \\
(\mathbf{n}=\mathbf{2 0})\end{array}$ & Significance \\
\hline Galvanic skin response $(\mathrm{mS})$ & & & \\
Dominant/non-hemiparetic & $1.13 \pm 0.90$ & $1.14 \pm 1.38$ & $\mathrm{NS}(\mathrm{p}=0.375)$ \\
Non-dominant/hemiparetic & $0.49 \pm 0.41$ & $0.11 \pm 0.25$ & $\mathrm{p}=0.001$ \\
\hline Skin temperature change $\left({ }^{\circ} \mathrm{C}\right)$ & $0.70 \pm 0.29$ & $0.45 \pm 0.41$ & $\mathrm{p}=0.030$ \\
\hline Electromyography $(\mathrm{mV})$ & $1.36 \pm 3.09$ & $-0.81 \pm 1.08$ & $\mathrm{p}=0.003$ \\
\hline
\end{tabular}

Results are expressed in terms of mean and standard deviation.

\section{Figure 3. Significant differences between groups in neurophysiological responses}
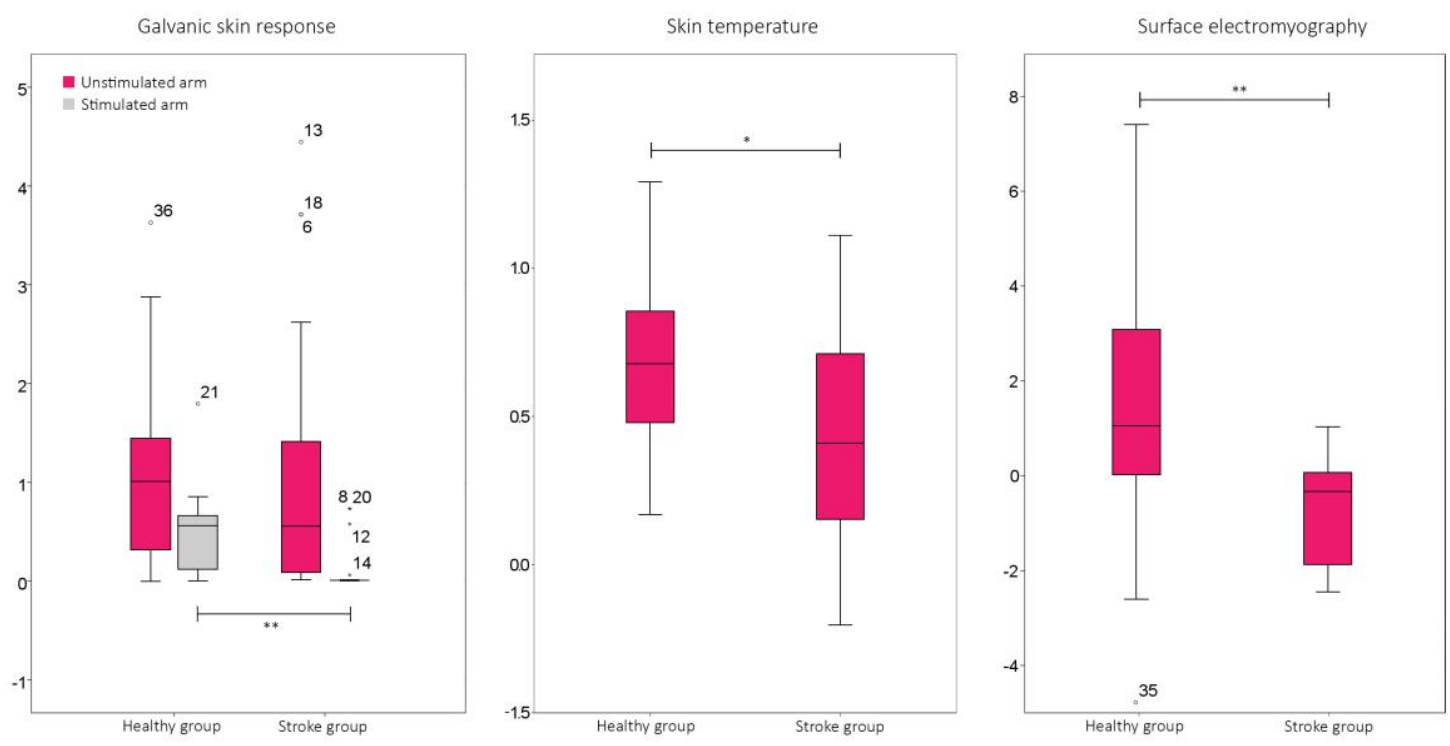

The picture shows a box and whisker plot of galvanic skin response, the skin temperature, and the surface electromyography. *: $p<0.05 ; * *: p<0.001$. 


\section{Interaction between embodiment and neurophysiological data}

Weak but statistically significant correlations were found between body-ownership and the GSR in the stimulated hand and the sEMG, and between localization and the GSR in the unstimulated hand (Table 5). A tendency towards signification was also detected between body-ownership and the GSR in the unstimulated hand, and between the agency in the stimulated hand. No other significant interactions were found.

Table 5. Interaction between embodiment and neurophysiological data

\begin{tabular}{lccc}
\hline & Body-ownership & Localization & Agency \\
\hline Galvanic skin response & & & \\
Dominant/non-hemiparetic & $\mathrm{r}=0.307$, & $\mathrm{r}=0.426$, & $\mathrm{r}=0.119$, \\
& $\mathrm{NS}(\mathrm{p}=0.051)$ & $\mathrm{p}=0.005$ & $\mathrm{NS}(\mathrm{p}=0.460)$ \\
Non-dominant/hemiparetic & $\mathrm{r}=-0.334$, & $\mathrm{r}=0.100$, & $\mathrm{r}=-0.301$, \\
& $\mathrm{p}=0.033$ & $\mathrm{NS}(\mathrm{p}=0.535)$ & $\mathrm{NS}(\mathrm{p}=0.056)$ \\
\hline Skin temperature change & $\mathrm{r}=0.102$, & $\mathrm{r}=0.158$, & $\mathrm{r}=0.244$, \\
& $\mathrm{NS}(\mathrm{p}=0.527)$ & $\mathrm{NS}(\mathrm{p}=0.323)$ & $\mathrm{NS}(\mathrm{p}=0.124)$ \\
\hline Electromyography & $\mathrm{r}=-0.314$, & $\mathrm{r}=0.027$, & $\mathrm{r}=-0.227$, \\
& $\mathrm{p}=0.046$ & $\mathrm{NS}(\mathrm{p}=0.865)$ & $\mathrm{NS}(\mathrm{p}=0.154)$ \\
\hline
\end{tabular}

NS: non-significant

\section{Discussion}

In this study, the subjective and neurophysiological responses of healthy individuals and hemiparetic individuals with stroke after the RHI were collected and compared. This is the first attempt to simultaneously assess different embodiment subcomponents (bodyownership, localization, and agency) and different neurophysiological measures (GSR, skin temperature, and sEMG) and the interaction between them in clinically controlled individuals with stroke. Our results showed that, compared to healthy participants, individuals with stroke experienced stronger body-ownership and agency and exhibited smaller increase of the GSR and the skin temperature, and reduced sEMG activity in the stimulated hand. Possible plasticity of the body schema of participants with stroke and a pathological increased predominance of the visual input over proprioception could have promoted the results in the embodiment subcomponents. A possible sudomotor dysregulation and the previously evidenced role of the premotor cortex in this process could explain the different neurophysiological responses, which did not evidence a clear relationship between them besides a weak relationship between body-ownership and GSR and sEMG, and between localization and GSR.

\section{Differences between groups}

\section{Embodiment}

The reported sense of body-ownership elicited by the experiment in healthy participants is supported by previous research. Earlier studies using similar versions of the questionnaire used in our study showed reports of body-ownership that ranged from 53\% (Petkova \& Ehrsson, 2009) to 78\% (Kalckert \& Ehrsson, 2014) during analogous 
visuotactile synchronous conditions. The significant stronger effect detected for participants with stroke contradicts a controlled study involving healthy participants (Zeller et al., 2011). However, differences in the sample (no reports on motor impairment), the procedure, and the measuring tools could explain this difference. In contrast, our results are in line with more recent reports. A study involving hemiplegic individuals post-stroke showed that stimulation of the affected hand elicited stronger illusion than stimulation of the less affected hand, which was attributed to a disruption of the normal integration between afferent and efferent signals (Burin et al., 2015). A previous exploratory study with a subject with hand disownership after stroke had also shown the same results (van Stralen et al., 2013). Interestingly, in the latter study visual exposure to the rubber hand was reported to be sufficient to elicit strong feelings of ownership, which highlights the role of the visual input in the experiment. In fact, visual inspection of a body part has been shown to enhance the detection of somatosensory stimuli, regardless of proprioceptive orienting (Tipper et al., 1998). In another study involving a participant post-stroke with unilateral spatial neglect, the experiment was reported to temporarily improve participant's performance in neglect tests (Kitadono \& Humphreys, 2007), which was attributed to a possible shift in the participant's sense of midline. Even though there is very limited research about how stroke can affect embodiment or the body schema, clinical reports of post-stroke individuals suffering from disorders of body representation and illusory own body perceptions could highlight the effects of this pathology in these mechanisms. We hypothesize that the high reports of body-ownership in participants with stroke could have been facilitated by a body schema plasticity promoted by the brain lesion and its resulting motor limitations (Frederiks, 1969). This condition could have allowed the external limb to be incorporated (thus promoting a reconfiguration of the body schema) more easily. Although the neural signatures of this mechanism still remain unknown, recent studies have evidenced the role of the premotor cortex of both hemispheres in embodiment of body parts (Arzy et al., 2006; Bekrater-Bodmann et al., 2014; Gentile et al., 2015; Limanowski \& Blankenburg, 2015; Petkova et al., 2011), also after stroke (Zeller et al., 2011). Interestingly, similar sensorimotor mechanisms have been shown to be recruited during the RHI and motor imagery (Ionta, Sforza, Funato, \& Blanke, 2013), and to elicit overlapped brain networks (Evans \& Blanke, 2013), including the premotor cortex (Gerardin et al., 2000; Ionta, Ferretti, Merla, Tartaro, \& Romani, 2010). The similarity of these networks with those active during motor execution (Grezes, Armony, Rowe, \& Passingham, 2003; Jeannerod, 2001) could explain that a breakdown of the network could have consequences to motor function, which in turn can affect the body-schema, and embodiment. In our study, cerebral infarctions in participants with ischemic stroke, all of them anterior, could have predominantly damaged this brain network, which could have promoted plastic alterations in their body schema.

Our results also suggest that body-ownership could be an attention-driven mechanism that evidences a predominance of the visual input over the proprioception in this effect. This could explain the fact that even though some participants had diminished tactile sensation, according to the results in the Nottingham Sensory 
Assessment, their reports about the effects of the experiment were as vivid as in other participants. This could also explain how body-ownership has been elicited in absence of tactile stimulation (van Stralen et al., 2013) and how viewing the body in a mirror can improve body-ownership in somatoparaphrenia (Jenkinson, Haggard, Ferreira, \& Fotopoulou, 2013). The visual influence on cortical motor areas has been shown to occur even with misalignment of the visual feedback (Touzalin-Chretien, Ehrler, \& Dufour, 2010), despite this incongruence disrupts motor execution (Wasaka \& Kakigi, 2012). Interestingly, the effectiveness of visual enhancement of touch depends on subjects' tactile acuity, improving tactile performance in subjects with lower tactile sensitivity (Serino, Farnè, Rinaldesi, Haggard, \& Làdavas, 2007), which could also evidence the visual dominance over proprioception in individuals with stroke. These hypotheses are consistent with previous results in neglect. Reconfiguration of the body schema through cueing attention could have shifted the egocentric representation of space to the left, consequently reducing neglect (Kitadono \& Humphreys, 2007).

Even though the RHI experiment isolates body-ownership in the absence of movement and efferent information, participants with stroke also reported to have felt agency over the rubber hand to a greater extent than healthy participants. Unfortunately, there are no previous reports on agency in stroke survivors. However, in a similar way to body-ownership, we hypothesize that the brain injury, together with its derived motor impairments could explain these results. Neuroimaging studies would be necessary to confirm the lesions of the premotor cortex and the involvement of this area in our sample, but if so, it could support the hypothesis that the premotor cortex not only encodes the body pose but also some control of proximal movements (Graziano \& Cooke, 2006; Graziano, Taylor, \& Moore, 2002). In spite of the fact that bodyownership and agency have been postulated as qualitatively different experiences (Tsakiris et al., 2010), this relationship, still on debate (Ma \& Hommel, 2015), could be more tangled in concomitant alterations to the body schema.

\section{Electromyography}

The premotor cortex, which has been evidenced as one of the brain areas involved in RHI, is also thought to be involved in the planning and execution of movements. It projects in to primary motor cortex and to the spinal cord, and receives somatosensory and visual input. Furthermore, it has been shown that the premotor cortex plays a role in orienting the body and readying the postural muscles for forthcoming movements (Rosenbaum, 2010). In addition, the ventral premotor cortex of macaques, lately named as polysensory zone (Graziano \& Cooke, 2006), has been suggested to contain a somatotopic representation of the arms, hands, face, and mouth, which even responds when a visual stimulus is placed in the region of space near the tactile receptive field (Gentilucci et al., 1988). This may imply that the premotor cortex could encode a representation of not only some body parts but also of the peripersonal space, which could be useful to anchor the world in relation to the body and enable rapid responses to threats and for hunting (Graziano et al., 2002). We speculate that the sense of bodyownership elicited by the experiment could have promoted a reconfiguration of the body 
schema, and consequently of the peripersonal space. The involvement of the premotor cortex in this mechanism, evidenced by previous studies, could also have had an unknown inhibitory effect that decreased muscle activity in individuals with stroke, who could present abnormally increased muscle activity, while having a null effect on heathy participants. Interestingly, lesions of premotor cortex have been reported to cause hypertonicity (Mukherjee \& Chakravarty, 2010).

Further neuroimaging studies involving participants with stroke should confirm the link between the damaged areas and the muscle tone. If true, even though the effects detected in our study could be temporary or clinically irrelevant, these findings could provide more insights about disorders affecting the upper motor neurons, such as spastic dystonia. It manifests as muscle overactivity at rest without a primary triggering factor, which causes abnormal position of some body parts (Denny-Brown, 1966) and poses a major cause of disability with only palliative treatments (symptoms are usually alleviated with drugs as baclofen, diazepam, or tizandine) with strong side effects. Although the clinical implication of these findings should be explored in future studies, since the underlying mechanisms of RHI have been recently linked to those from mirror-therapy and motor imagery (Moseley, Gallace, \& Spence, 2008), and dynamic changes in perceived ownership by the RHI have been suggested to be trait-like (Bekrater-Bodmann, Foell, Diers, \& Flor, 2012), if these hypotheses are correct, the effects of the RHI could be used to predict the effectiveness of these treatments in stroke individuals.

\section{Galvanic skin response}

The increase in the GSR in the unstimulated hand during the experiment is supported by previous findings in the unstimulated hand across different populations. Similar results have been reported in healthy subjects (Armel \& Ramachandran, 2003; Ma \& Hommel, 2013), in upper limb amputees (Ehrsson et al., 2008), and in subjects with complex regional pain syndrome (Reinersmann et al., 2013). However, there is no previous research on the effects of the experiment on the stimulated hand. This lack of evidence is possibly motivated by limitations in the instrumentation used to measure the GSR. Common devices, as the one used in this study to explore the unstimulated hand, usually have two sensors that are attached to the fingers, thus impeding their stroking. In our study, the use of a bracelet allowed us to simultaneously measure the response in the stimulated hand, evidencing a less relevant increase of the GSR in this side during the experiment, significantly smaller in participants with stroke than in the healthy group. Nonetheless, even though this effect could be ascribed to the effects of the experiment, it could have also been motivated by a sudomotor dysregulation derived from the brain injury, which has been reported after cerebrovascular diseases, particularly after ischemic stroke in acute and chronic stages (Korpelainen, Sotaniemi, \& Myllyla, 1999; Meyer, Strittmatter, Fischer, Georg, \& Schmitz, 2004; Muslumanoglu, Akyuz, Aki, Karsidag, \& Us, 2002). Stroke has been reported to suppress the reflex activity of the sympathetic nervous system (Korpelainen, Tolonen, 
Sotaniemi, \& Myllyla, 1993), which can cause asymmetric responses after a focal brain lesion (Linden \& Berlit, 1995).

Regardless of the cause, the increase in both hands exceeded the proposed threshold of relevance (Armel \& Ramachandran, 2003), thus supporting the fact that the RHI elicits neurocognitive mechanisms that trigger the GSR of both hands in both healthy subjects and individuals with stroke. Again, we speculate that the involvement of the premotor cortex in the RHI could have elicited electrodermal activity that caused variations in the GSR. The close connection between the pyramidal (corticospinal) fibers for the transmission of skeletal muscle impulse and sudomotor (corticopontine) fibers could explain this effect. Interestingly, it has been posed that the combined striatal and premotor cortical origins of electrodermal activity can be viewed together as a single premotor electrodermal component and a concomitant of nonpyramidal motor system activity (Boucsein, 2012). Whether the increase of the GSR is caused by a reconfiguration of the body schema (and the consequent involvement of the premotor cortex) or by a threat to a human-like arm is still controversial (Ma \& Hommel, 2013).

\section{Skin temperature}

Different measures of skin temperature variation, generally measured with infrared thermometers, have been used as neurophysiological correlates of the RHI. As opposed to the increase in the skin temperature revealed in our study, it was initially postulated that the illusion evokes a limb-specific decrease that correlates with the strength of the illusion (Moseley, Olthof, et al., 2008). Furthermore, the relationship between temperature and body-ownership has been suggested to be causal: cooling down the participant's hand has been reported to increase the strength of the experiment and vice versa (Kammers et al., 2011). However, changes in temperature between synchronous and asynchronous stimulation through finger touches have shown limitations in discriminating between experimental variations of the experiment (Hohwy \& Paton, 2010). In addition, a study involving individuals with schizophrenia showed less compelling results. Only when the right hand was stimulated, a significant heating of the unstimulated hand and cooling of the stimulated hand were detected (Thakkar et al., 2011). Another study found no evidence for hand cooling in two experiments involving healthy subjects using a robot-based stroking procedure and only a non-significant difference between hands during manual stroking (Rohde et al., 2013). These results were similar in a series of experiments evaluating the stroking velocity, side of the stimulation, and the material, concluding that skin temperature could be a less robust outcome measure of the RHI (van Stralen et al., 2014).

As for sEMG and GSR, we conjecture that the involvement of the premotor cortex in the RHI could have promoted the variations in the skin temperature. Electrical stimulation (and even removal) of premotor cortical areas has shown excitatory as well as inhibitory sudorisecretory influences (Boucsein, 2012), which could explain the different results reported by preliminary studies (Moseley, Olthof, et al., 2008; Rohde et al., 2013). In addition, temperature regulation might also result from changes in arousal and attentional drive (Thakkar et al., 2011), which could be key factors to promote 
body-ownership. We hypothesized that these factors can cause conflicting effects which can mask each other. These effects, together with possible non-stabilized temperature conditions in previous studies, could have led to misleading interpretation of the results, and should be carefully addressed in further studies.

\section{Interaction between embodiment and neurophysiological data}

Although the neurophysiological measures showed different responses in healthy and post-stroke subjects, these measures failed to show convergent validity with nearly all of the subjective evaluations of embodiment. Although the only significant correlations, which were found in the body-ownership and the localization, were weak, they might evidence some effects worth to discuss. First, the correlation found between bodyownership and the GSR in the stimulated (statistically significant) and unstimulated hand (almost statistically significant) could evidence a relationship between bodyownership and the sudomotor activity, in line with previous reports (Armel \& Ramachandran, 2003). Interestingly, our results appeared to show that this interaction is inverse in both arms: greater body-ownership is associated to a decrease in GSR in the stimulated hand and an increase in the unstimulated hand. Second, sEMG seemed to be associated to body-ownership, in such a way that greater embodiment was linked to greater decrease or relaxation of the muscular activity. Finally, the greatest but still weak correlation, which was detected between localization and the GSR in the unstimulated hand, could indicate that the body schema reorientation reflected by the localization might influence sudomotor activity, as has been previously posed for bodyownership (Armel \& Ramachandran, 2003; D'Alonzo \& Cipriani, 2012; Ehrsson et al., 2008; Ma \& Hommel, 2013). Unfortunately, since this is the first study to investigate multiple embodiment constructs and neurophysiological variables, comparison with other reports was not possible. Future studies should confirm these effects (and, if so, clarify whether the muscular relaxation and the sudomotor activity are cause or consequence of the elicited body-ownership and localization, respectively) or attribute them to the multiple analysis performed, rather than evidencing a linear relationship between the neurophysiological measures and the embodiment mechanisms.

In any event, our results and all of the contradicting reports of the previous studies that individually explored neurophysiological correlates of the RHI suggest that it would be misleading to assume a one-to-one relationship between the subjective perceptions of embodiment and the physiological responses elicited during the RHI. Although, as mentioned, there are no previous attempts to study this interaction, we argue that this relationship could be far too complex to be captured by a linear correlation and, moreover, it could be affected by different factors that may vary across and within studies.

\section{Limitations}

The limitations of this study must be carefully considered when analyzing the results. First, the sample size (41 participants) can be considered small, though it is similar to or larger than those typically involved in state-of-the-art interventions (Lloyd et al., 2013; 
Salomon et al., 2013). Second, all of the participants were attending a neurorehabilitation program, which could affect in an unknown fashion to the results in the experiment. Third, no records of the proprioceptive drift were available. The residual motor impairment even in the less affected (unstimulated) arm of participants with stroke prevented this measure to be registered reliably. Fourth, no records of the skin temperature in the unstimulated hand are available, which does not allow the comparison between temperature variations in both hands. Fifth, in a similar manner, the different devices used for registering the GSR prevented the study of limb-specific responses. Finally, no magnetic resonance imaging-based volumetric measurements of the premotor area are available, which prevented volumes to be correlated with neurophysiological, neuropathological, and neuropsychological findings of our study. However, the homogeneity of the sample in demographic and clinical variables (only individuals without neglect and asomatognosia were included), the assessment of the motor, sensory, and cognitive condition of participants with stroke, the examination of different sub-components of embodiment, and the concurrent exploration of different neurophysiological variables and their interactions, support the results of our study.

\section{Conclusions}

In this study, the subjective and neurophysiological responses of healthy individuals and hemiparetic individuals with stroke after the RHI were collected and compared. The experiment elicited stronger body-ownership and agency in hemiparetic individuals with stroke than in healthy individuals. Possible body schema plasticity and a pathological increased predominance of the visual input over proprioception could have promoted the results. Both groups showed different neurophysiological responses to the experiment. Individuals with stroke exhibited smaller increase of the GSR and the skin temperature, and reduced sEMG activity in the stimulated hand. A possible suppression of the reflex activity of the sympathetic nervous system caused by the brain injury and the involvement of the premotor cortex in the reconfiguration of the body schema promoted by the mechanisms of body-ownership could have motivated the main differences in the neurophysiological responses. Analyses of the interactions between subjective and neurophysiological responses were inconclusive, while they could point to a weak relationship between body-ownership and sEMG and GSR, and between localization and GSR. These results could evidence a body schema plasticity promoted by brain lesions and support the role of the premotor cortex in this mechanism.

\section{Acknowledgments}

The authors wish to thank to the staff and patients of the Servicio de Neurorrehabilitación y Daño Cerebral de los Hospitales NISA for their participation in this study. This work was supported by Ministerio de Economía y Competitividad of Spain (Project NeuroVR, TIN2013-44741-R and Project REACT, TIN2014-61975EXP), by Universitat Politècnica de València (Grant PAID-10-14), and by the "CIBER of Physiopathology of Obesity and Nutrition, an initiative of ISCIII". The authors have no conflict of interest to declare. 


\section{References}

Aimola Davies, A. M., \& White, R. C. (2013). A sensational illusion: vision-touch synaesthesia and the rubber hand paradigm. Cortex; a journal devoted to the study of the nervous system and behavior, 49(3), 806-818.

Armel, K. C., \& Ramachandran, V. S. (2003). Projecting sensations to external objects: evidence from skin conductance response. Proceedings. Biological sciences / The Royal Society, 270(1523), 1499-1506.

Arzy, S., Overney, L. S., Landis, T., \& Blanke, O. (2006). Neural mechanisms of embodiment: Asomatognosia due to premotor cortex damage. Archives of Neurology, 63(7), 1022-1025.

Bekrater-Bodmann, R., Foell, J., Diers, M., \& Flor, H. (2012). The perceptual and neuronal stability of the rubber hand illusion across contexts and over time. Brain research, 1452, 130-139.

Bekrater-Bodmann, R., Foell, J., Diers, M., Kamping, S., Rance, M., Kirsch, P., . . . Flor, H. (2014). The importance of synchrony and temporal order of visual and tactile input for illusory limb ownership experiences - an FMRI study applying virtual reality. PloS one, 9(1), e87013.

Berlucchi, G., \& Aglioti, S. (1997). The body in the brain: neural bases of corporeal awareness. Trends in neurosciences, 20(12), 560-564.

Bohannon, R. W., \& Smith, M. B. (1987). Interrater reliability of a modified Ashworth scale of muscle spasticity. Physical therapy, 67(2), 206-207.

Botvinick, M., \& Cohen, J. (1998). Rubber hands $/$ feel/' touch that eyes see. Nature, 391(6669), 756-756.

Boucsein, W. (2012). Electrodermal Activity (pp. 1-86): Springer US.

Burin, D., Livelli, A., Garbarini, F., Fossataro, C., Folegatti, A., Gindri, P., \& Pia, L. (2015). Are movements necessary for the sense of body ownership? Evidence from the rubber hand illusion in pure hemiplegic patients. PloS one, 10(3), $\mathrm{e} 0117155$.

Clancy, E. A., Morin, E. L., \& Merletti, R. (2002). Sampling, noise-reduction and amplitude estimation issues in surface electromyography. Journal of electromyography and kinesiology : official journal of the International Society of Electrophysiological Kinesiology, 12(1), 1-16.

Corbett, A., \& Shah, S. (1996). Body Scheme Disorders following Stroke and Assessment in Occupational Therapy. The British Journal of Occupational Therapy, 59(7), 325-329.

D'Alonzo, M., \& Cipriani, C. (2012). Vibrotactile sensory substitution elicits feeling of ownership of an alien hand. PloS one, 7(11), e50756.

de Vignemont, F. (2011). Embodiment, ownership and disownership. Consciousness and cognition, 20(1), 82-93.

Denny-Brown, D. (1966). The cerebral control of movement. Liverpool: Liverpool University Press.

Ehrsson, H. H., Rosén, B., Stockselius, A., Ragnö, C., Köhler, P., \& Lundborg, G. (2008). Upper limb amputees can be induced to experience a rubber hand as their own (Vol. 131).

Evans, N., \& Blanke, O. (2013). Shared electrophysiology mechanisms of body ownership and motor imagery. NeuroImage, 64, 216-228.

Folstein, M. F., Folstein, S. E., \& McHugh, P. R. (1975). "Mini-mental state". A practical method for grading the cognitive state of patients for the clinician. Journal of psychiatric research, 12(3), 189-198. 
Frederiks, J. A. M. (1969). Disorders of the body schema. In P. J. Vinken \& G. W. Bruyn (Eds.), Handbook of clinical neurology (Vol. 4, pp. 207-240). Amsterdam: Elsevier.

Gentile, G., Bjornsdotter, M., Petkova, V. I., Abdulkarim, Z., \& Ehrsson, H. H. (2015). Patterns of neural activity in the human ventral premotor cortex reflect a wholebody multisensory percept. NeuroImage, 109, 328-340.

Gentilucci, M., Fogassi, L., Luppino, G., Matelli, M., Camarda, R., \& Rizzolatti, G. (1988). Functional organization of inferior area 6 in the macaque monkey. I. Somatotopy and the control of proximal movements. Experimental brain research, 71(3), 475-490.

Gerardin, E., Sirigu, A., Lehericy, S., Poline, J. B., Gaymard, B., Marsault, C., . . . Le Bihan, D. (2000). Partially overlapping neural networks for real and imagined hand movements. Cerebral cortex, 10(11), 1093-1104.

Graziano, M. S., \& Cooke, D. F. (2006). Parieto-frontal interactions, personal space, and defensive behavior. Neuropsychologia, 44(13), 2621-2635.

Graziano, M. S., Taylor, C. S., \& Moore, T. (2002). Complex movements evoked by microstimulation of precentral cortex. Neuron, 34(5), 841-851.

Grezes, J., Armony, J. L., Rowe, J., \& Passingham, R. E. (2003). Activations related to "mirror" and "canonical" neurones in the human brain: an fMRI study. NeuroImage, 18(4), 928-937.

Guterstam, A., Bjornsdotter, M., Gentile, G., \& Ehrsson, H. H. (2015). Posterior cingulate cortex integrates the senses of self-location and body ownership. Current biology : CB, 25(11), 1416-1425.

Haggard, P., \& Wolpert, D. M. (2005). Disorders of body schema. In H. J. Freund, M. Jeannerod, M. Hallett \& R. Leiguarda (Eds.), Higher-order motor disorders: from neuroanatomy and neurobiology to clinical neurology (pp. 261-271). Oxford: Oxford University Press.

Hohwy, J., \& Paton, B. (2010). Explaining away the body: experiences of supernaturally caused touch and touch on non-hand objects within the rubber hand illusion. PloS one, 5(2), e9416.

Huis In 't Veld, E. M., van Boxtel, G. J., \& de Gelder, B. (2014). The Body Action Coding System II: muscle activations during the perception and expression of emotion. Frontiers in behavioral neuroscience, 8, 330.

Ionta, S., Ferretti, A., Merla, A., Tartaro, A., \& Romani, G. L. (2010). Step-by-step: the effects of physical practice on the neural correlates of locomotion imagery revealed by fMRI. Human brain mapping, 31(5), 694-702.

Ionta, S., Sforza, A., Funato, M., \& Blanke, O. (2013). Anatomically plausible illusory posture affects mental rotation of body parts. Cognitive, affective \& behavioral neuroscience, 13(1), 197-209.

Jeannerod, M. (2001). Neural simulation of action: a unifying mechanism for motor cognition. NeuroImage, 14(1 Pt 2), S103-109.

Jenkinson, P. M., Haggard, P., Ferreira, N. C., \& Fotopoulou, A. (2013). Body ownership and attention in the mirror: insights from somatoparaphrenia and the rubber hand illusion. Neuropsychologia, 51(8), 1453-1462.

Kalckert, A., \& Ehrsson, H. (2012). Moving a rubber hand that feels like your own: dissociation of ownership and agency. Frontiers in Human Neuroscience, 6.

Kalckert, A., \& Ehrsson, H. H. (2014). The moving rubber hand illusion revisited: comparing movements and visuotactile stimulation to induce illusory ownership. Consciousness and cognition, 26, 117-132. 
Kammers, M. P., de Vignemont, F., Verhagen, L., \& Dijkerman, H. C. (2009). The rubber hand illusion in action. Neuropsychologia, 47(1), 204-211.

Kammers, M. P. M., Rose, K., \& Haggard, P. (2011). Feeling numb: Temperature, but not thermal pain, modulates feeling of body ownership. Neuropsychologia, 49(5), 1316-1321.

Kelly-Hayes, M., Beiser, A., Kase, C. S., Scaramucci, A., D'Agostino, R. B., \& Wolf, P. A. (2003). The influence of gender and age on disability following ischemic stroke: the Framingham study. Journal of stroke and cerebrovascular diseases : the official journal of National Stroke Association, 12(3), 119-126.

Kitadono, K., \& Humphreys, G. W. (2007). Short-term Effects of the 'Rubber Hand' Illusion on Aspects of Visual Neglect. Neurocase, 13(4), 260-271.

Kopp, B., Kunkel, A., Flor, H., Platz, T., Rose, U., Mauritz, K. H., . . Taub, E. (1997). The Arm Motor Ability Test: reliability, validity, and sensitivity to change of an instrument for assessing disabilities in activities of daily living. Archives of physical medicine and rehabilitation, 78(6), 615-620.

Korpelainen, J. T., Sotaniemi, K. A., \& Myllyla, V. V. (1999). Autonomic nervous system disorders in stroke. Clinical autonomic research : official journal of the Clinical Autonomic Research Society, 9(6), 325-333.

Korpelainen, J. T., Tolonen, U., Sotaniemi, K. A., \& Myllyla, V. V. (1993). Suppressed sympathetic skin response in brain infarction. Stroke; a journal of cerebral circulation, 24(9), 1389-1392.

Legrand, D. (2006). The Bodily Self: The Sensori-Motor Roots of Pre-Reflective SelfConsciousness. Phenom Cogn Sci, 5(1), 89-118.

Limanowski, J., \& Blankenburg, F. (2015). Network activity underlying the illusory self-attribution of a dummy arm. Human brain mapping, 36(6), 2284-2304.

Lincoln, N. B., Jackson, J. M., \& Adams, S. A. (1998). Reliability and Revision of the Nottingham Sensory Assessment for Stroke Patients. Physiotherapy, 84(8), 358365.

Linden, D., \& Berlit, P. (1995). Sympathetic skin responses (SSRs) in monofocal brain lesions: topographical aspects of central sympathetic pathways. Acta neurologica Scandinavica, 91(5), 372-376.

Longo, M. R., Schüür, F., Kammers, M. P. M., Tsakiris, M., \& Haggard, P. (2008). What is embodiment? A psychometric approach. Cognition, 107(3), 978-998.

Lloyd, D. M., Gillis, V., Lewis, E., Farrell, M. J., \& Morrison, I. (2013). Pleasant touch moderates the subjective but not objective aspects of body perception. Frontiers in behavioral neuroscience, 7 .

Ma, K., \& Hommel, B. (2013). The virtual-hand illusion: effects of impact and threat on perceived ownership and affective resonance. Frontiers in psychology, 4, 604.

Ma, K., \& Hommel, B. (2015). The role of agency for perceived ownership in the virtual hand illusion. Consciousness and cognition, 36, 277-288.

Meyer, S., Strittmatter, M., Fischer, C., Georg, T., \& Schmitz, B. (2004). Lateralization in autonomic dysfunction in ischemic stroke involving the insular cortex. Neuroreport, 15(2), 357-361.

Moseley, G. L., Olthof, N., Venema, A., Don, S., Wijers, M., Gallace, A., \& Spence, C. (2008). Psychologically induced cooling of a specific body part caused by the illusory ownership of an artificial counterpart. Proceedings of the National Academy of Sciences of the United States of America, 105(35), 13169-13173.

Moseley, L. G., Gallace, A., \& Spence, C. (2008). Is mirror therapy all it is cracked up to be? Current evidence and future directions. Pain, 138(1), 7-10. 
Mukherjee, A., \& Chakravarty, A. (2010). Spasticity mechanisms - for the clinician. Frontiers in neurology, 1, 149.

Muslumanoglu, L., Akyuz, G., Aki, S., Karsidag, S., \& Us, O. (2002). Evaluation of autonomic nervous system functions in post-stroke patients. American journal of physical medicine \& rehabilitation / Association of Academic Physiatrists, 81(10), 721-725.

Ocklenburg, S., Ruther, N., Peterburs, J., Pinnow, M., \& Gunturkun, O. (2011). Laterality in the rubber hand illusion. Laterality, 16(2), 174-187.

Oldfield, R. C. (1971). The assessment and analysis of handedness: the Edinburgh inventory. Neuropsychologia, 9(1), 97-113.

Petkova, V. I., Bjornsdotter, M., Gentile, G., Jonsson, T., Li, T. Q., \& Ehrsson, H. H. (2011). From part- to whole-body ownership in the multisensory brain. Current biology : $C B, 21(13), 1118-1122$.

Petkova, V. I., \& Ehrsson, H. H. (2009). When Right Feels Left: Referral of Touch and Ownership between the Hands. PloS one, 4(9), e6933.

Ramakonar, H., Franz, E. A., \& Lind, C. R. (2011). The rubber hand illusion and its application to clinical neuroscience. Journal of clinical neuroscience : official journal of the Neurosurgical Society of Australasia, 18(12), 1596-1601.

Reinersmann, A., Landwehrt, J., Krumova, E. K., Peterburs, J., Ocklenburg, S., Gunturkun, O., \& Maier, C. (2013). The rubber hand illusion in complex regional pain syndrome: preserved ability to integrate a rubber hand indicates intact multisensory integration. Pain, 154(9), 1519-1527.

Rohde, M., Wold, A., Karnath, H. O., \& Ernst, M. O. (2013). The human touch: skin temperature during the rubber hand illusion in manual and automated stroking procedures. PloS one, 8(11), e80688.

Romero, M., Sanchez, A., Marin, C., Navarro, M. D., Ferri, J., \& Noe, E. (2012). Clinical usefulness of the Spanish version of the Mississippi Aphasia Screening Test (MASTsp): validation in stroke patients. Neurologia, 27(4), 216-224.

Rosenbaum, D. A. (2010). Physiological Foundations. In D. A. Rosenbaum (Ed.), Human Motor Control (Second Edition) (pp. 43-91). San Diego: Academic Press.

Salomon, R., Lim, M., Pfeiffer, C., Gassert, R., \& Blanke, O. (2013). Full body illusion is associated with widespread skin temperature reduction. Frontiers in behavioral neuroscience, 7.

Serino, A., Farnè, A., Rinaldesi, M. L., Haggard, P., \& Làdavas, E. (2007). Can vision of the body ameliorate impaired somatosensory function? Neuropsychologia, 45(5), 1101-1107.

Thakkar, K. N., Nichols, H. S., McIntosh, L. G., \& Park, S. (2011). Disturbances in body ownership in schizophrenia: evidence from the rubber hand illusion and case study of a spontaneous out-of-body experience. PloS one, 6(10), e27089.

Tipper, S. P., Lloyd, D., Shorland, B., Dancer, C., Howard, L. A., \& McGlone, F. (1998). Vision influences tactile perception without proprioceptive orienting. Neuroreport, 9(8), 1741-1744.

Touzalin-Chretien, P., Ehrler, S., \& Dufour, A. (2010). Dominance of vision over proprioception on motor programming: evidence from ERP. Cerebral cortex, 20(8), 2007-2016.

Tsakiris, M. (2010). My body in the brain: A neurocognitive model of body-ownership. Neuropsychologia, 48(3), 703-712. 
Tsakiris, M., Hesse, M. D., Boy, C., Haggard, P., \& Fink, G. R. (2007). Neural signatures of body ownership: a sensory network for bodily self-consciousness. Cerebral cortex, 17(10), 2235-2244.

Tsakiris, M., Longo, M. R., \& Haggard, P. (2010). Having a body versus moving your body: Neural signatures of agency and body-ownership. Neuropsychologia, 48(9), 2740-2749.

van Stralen, H. E., van Zandvoort, M. J., Hoppenbrouwers, S. S., Vissers, L. M., Kappelle, L. J., \& Dijkerman, H. C. (2014). Affective touch modulates the rubber hand illusion. Cognition, 131(1), 147-158.

van Stralen, H. E., van Zandvoort, M. J., Kappelle, L. J., \& Dijkerman, H. C. (2013). The Rubber Hand Illusion in a patient with hand disownership. Perception, 42(9), 991-993.

Wasaka, T., \& Kakigi, R. (2012). Conflict caused by visual feedback modulates activation in somatosensory areas during movement execution. NeuroImage, 59(2), 1501-1507.

Zeller, D., Gross, C., Bartsch, A., Johansen-Berg, H., \& Classen, J. (2011). Ventral Premotor Cortex May Be Required for Dynamic Changes in the Feeling of Limb Ownership: A Lesion Study. The Journal of Neuroscience, 31(13), 4852-4857. 